\title{
A Geometry and Collimation Study of a Compton Backscatter Device for Inclusions Detection in Materials
}

\author{
Emerson M. Boldo ${ }^{1}$, Ana A. P. Prestes and Carlos R. Appoloni \\ Laboratório de Física Nuclear Aplicada \\ Departamento de Física - CCE \\ Universidade Estadual de Londrina \\ Rodovia Celso Garcia Cid, Pr 445 Km 380 \\ 86055-900 Londrina - PR, Brazil \\ E-mail: eboldo@gmail.com
}

\begin{abstract}
Compton backscattering of gamma rays is a nondestructive technique that can be used for material characterization and detection of defects and inclusions in materials. The methodology allows one-side inspection of large structures, is relatively inexpensive and can be portable. The concept is based on detection of backscattered radiation produced from a collimated beam aimed at the sample. The energy spectrum of backscattered photons can be used to determine local density perturbations. In this work we carried out a geometry and collimation study of the Compton backscatter device that consists of a $\varnothing 2 \mathrm{~mm}$ collimated ${ }^{241} \mathrm{Am}(100 \mathrm{mCi})$ gamma source and a high resolution CdTe semiconductor detector. Acrylic and plaster blocks with cylindrical holes and steel inclusions were used as samples. Collimators in the detector with $\emptyset 7$ $\mathrm{mm}, \varnothing 5 \mathrm{~mm}, \varnothing 3 \mathrm{~mm}$ and $\varnothing 1.5 \mathrm{~mm}$ were tested in two different scattering angles: $135^{\circ}$ and $150^{\circ}$. The results showed that the backscatter geometry of $135^{\circ}$ with the $\varnothing 7 \mathrm{~mm}$ collimator resulted in higher contrast in the intensity versus position profile without loss of spatial resolution. The tests on plaster blocks with steel rods inclusions suggest that, for a low energy and activity gamma source, the effects of beam attenuation are more decisive to the scattered intensity than increasing of material density. With the density contrast analysis, size and depth of steel rods can be determined.
\end{abstract}

XXXIV edition of the Brazilian Workshop on Nuclear Physics,

Foz de Iguaçu, Parana state, Brasil

5-10 June 2011

Speaker 


\section{Introduction}

Compton backscattering has the potential to be an attractive technique for detecting and positioning inclusions in materials. By using this technique, measurements can be performed on only one side of a sample, on untreated surfaces, and with good spatial resolution.

The technique is based on monitoring the scattering intensity in an inspection volume formed by the intersection of the solid angles of the source and detector. Under the Compton effect, the scattering intensity is approximately proportional to the density of the material. Larger inspection volumes enhance the detector count rate, which in turn reduces the measurement time. However, large inspection volumes decrease the resolution and density contrast, and this reduces the effectiveness of the technique in distinguishing between different materials within the sample.

The size of the inspection volume and the paths of the incident and scattered beams within the material being inspected may be changed using different scattering angles and collimation geometries. Therefore, there is a tradeoff between these variables when optimizing the experimental arrangement. In this work, a collimation and geometry study was carried out with the aim of determining the best experimental configuration to detect inclusions and voids in materials using a compact portable system based on the Compton scattering technique. Two different scattering angles $\left(135^{\circ}\right.$ and $\left.150^{\circ}\right)$ and four collimators with different apertures and materials were tested. Detection capacity, spatial resolution, and density contrast were the parameters considered when evaluating the chosen experimental geometry.

\section{Theoretical Background}

The number of photons scattered by the Compton effect depends on the electron density of the material analyzed. If $N_{A}$ is the Avogadro's number, $Z$ the target atomic number and $A$ its atomic mass, the number of electrons $/ \mathrm{cm}^{3}$ is given by:

$$
\rho_{e}=N_{A}\left(\frac{Z}{A}\right) \rho
$$

where $\rho$ represents physical density of the material (in $\mathrm{g} / \mathrm{cm}^{3}$ ). For non-hydrogenous materials $\mathrm{Z} / \mathrm{A} \approx 1 / 2$.

Adding the beam attenuation of the incident and scattered beam within the material, the number of photons that are subjected to scattering inside a well-defined volume $(V O L)$ and reach the detector can be given by [1]:

$$
I_{C}=C I_{0} V O L \rho_{e} \exp \left[-\mu\left(E_{i}\right) X_{i}\right] \exp \left[-\mu\left(E_{S}\right) X_{S}\right]+M_{C}
$$

where $C$ is a proportionality constant that includes solid angle, detector efficiency and the differential cross section per electron, $I_{0}$ is the incident intensity and $\rho_{e}$ is the average electron density within the inspection volume. Both exponential functions represent attenuation of the 
incident and scattered beam along the paths $X_{i}$ and $X_{S}$ within the material and $\mu\left(E_{i}\right)$ and $\mu\left(E_{S}\right)$ are the respective attenuation coefficients. Lastly, $M_{c}$ is the contribution of multiple scatterings.

In this work, we have defined density contrast $(D C)$ mathematically as:

$$
D C=\left(\frac{C_{\text {bulk }}-C_{\text {inclusion }}}{C_{\text {bulk }}}\right) \times 100 \%
$$

where $C_{b u l k}$ is the mean count values when the incident beam is only on the sample material and $C_{\text {inclusion }}$ represents mean count values registered by the detector when the incident beam intercepts an inclusion inside the sample. Contrast defines the ability of the system in distinguishing amongst materials of different densities.

\section{Materials and Methods}

The experimental setup used in this study consisted of an ${ }^{241} \mathrm{Am}(100 \mathrm{mCi})$ collimated source $(\varnothing 2 \mathrm{~mm})$ positioned at a distance of $100 \mathrm{~mm}$ from the sample and a high-resolution CdTe detector (model: Amptek X-123) placed on the same side of the source regarding the sample. These components were chosen in order to construct a lightweight, portable, and compact device. Two scattering angles were evaluated: $135^{\circ}$ and $150^{\circ}$. Four cylindrical detector collimators measuring $\varnothing 7 \times 30 \mathrm{~mm}, \varnothing 5 \times 15 \mathrm{~mm}, \varnothing 3 \times 15 \mathrm{~mm}$, and $\varnothing 1.5 \times 15$ were tested. The first collimator $(30 \mathrm{~mm})$ was made of brass, whereas the other three were made of aluminum.

Each sample used in this experiment consists of blocks of one of the following materials: acrylic $\left(\rho=1.17 \mathrm{~g} / \mathrm{cm}^{3}\right)$, aluminum $\left(\rho=2.72 \mathrm{~g} / \mathrm{cm}^{3}\right)$, plaster $\left(\rho=0.81 \mathrm{~g} / \mathrm{cm}^{3}\right)$, and concrete $(\rho=$ $\left.2.34 \mathrm{~g} / \mathrm{cm}^{3}\right)$. For the inclusion detection tests, a plaster block with steel bars $\left(\rho=7.68 \mathrm{~g} / \mathrm{cm}^{3}\right)$ that had diameters of $\varnothing 8 \mathrm{~mm}$ and $\varnothing 10 \mathrm{~mm}$ and were embedded $14 \mathrm{~mm}$ into the sample was used. The samples were mounted on a mobile support, which allows for lateral movement perpendicular to the incident beam in steps of $1 \mathrm{~mm}$ and $2 \mathrm{~mm}$. The detector, which had a builtin preamplifier, digital pulse processor, and multichannel analyzer, was connected to a notebook for data collection and analysis.

\section{Results}

Figure 1 (left) shows two source and detector configurations studied in this work. One of the configurations is such that the incident beam is perpendicular to the sample (Geometry 1). In the other (Geometry 2), the positions of the detector and source are changed such that the backscattered photons are captured in a direction perpendicular to the sample. The geometries were set such that the center of the inspection volume was located at a depth of $15 \mathrm{~mm}$ from the surface.

The net counts of the backscattered photons reaching the detector in the two geometries are shown in Fig. 1 (right). In Geometry 2, a larger portion of the inspection volume (VOL) is closer to the surface; therefore, the path traveled by the scattered photons within the material toward the detector is shorter. This explains the greater photon count in the case of Geometry 2. 
Greater counts imply shorter measurement times for obtaining statistically significant values during the experiment.

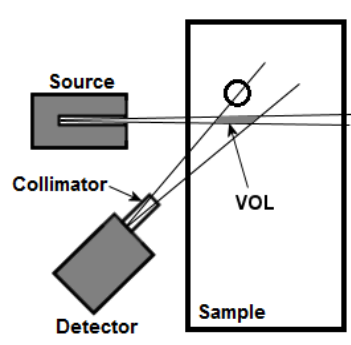

Geometry 01
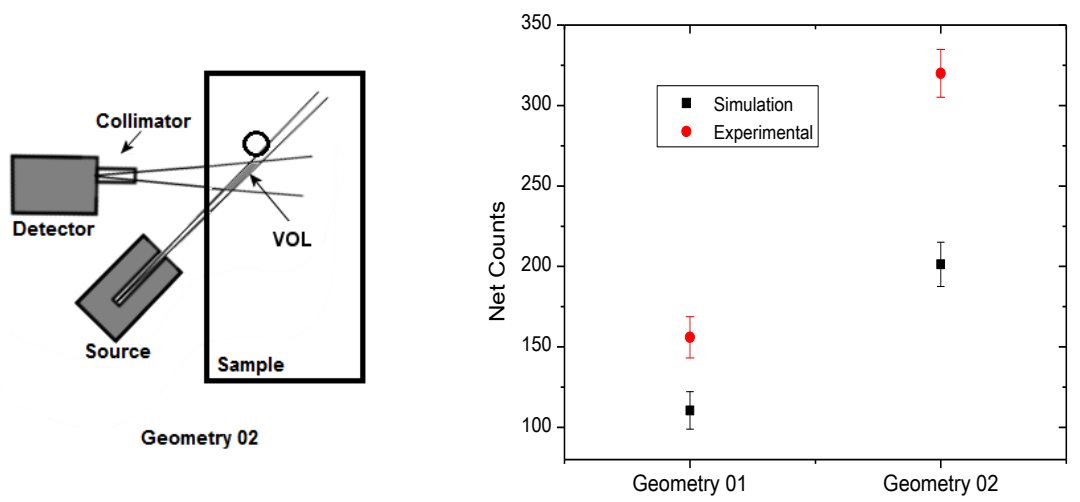

Figure 1. Two geometries of the experimental setup (left). Net counts obtained by the detector in the two geometries (right). Counting time: $1000 \mathrm{~s}$, scattering angle: $135^{\circ}$.

Even though Geometry 2 had the abovementioned advantages, it was not chosen because of certain practical considerations. As the inspection volume is oblique to the sample surface and its size along this direction depends mainly on detector collimation, eventually the inspection volume may reach a region of interest without the detector being exactly above it. Consequently, an external reference in the region of interest is not guaranteed, regardless of whether the region has an inclusion or a defect.

The beam direction in Geometry 1 may be viewed as an external reference. When the perpendicular beam crosses a region in the sample where the density varies, the detector immediately registers a change in the scattered photon count.

Figure 2 shows the results obtained when the four collimators were tested. Each point represents the mean value of five integrated counts of the Compton peak. The test was conducted for two angles and four different targets. 


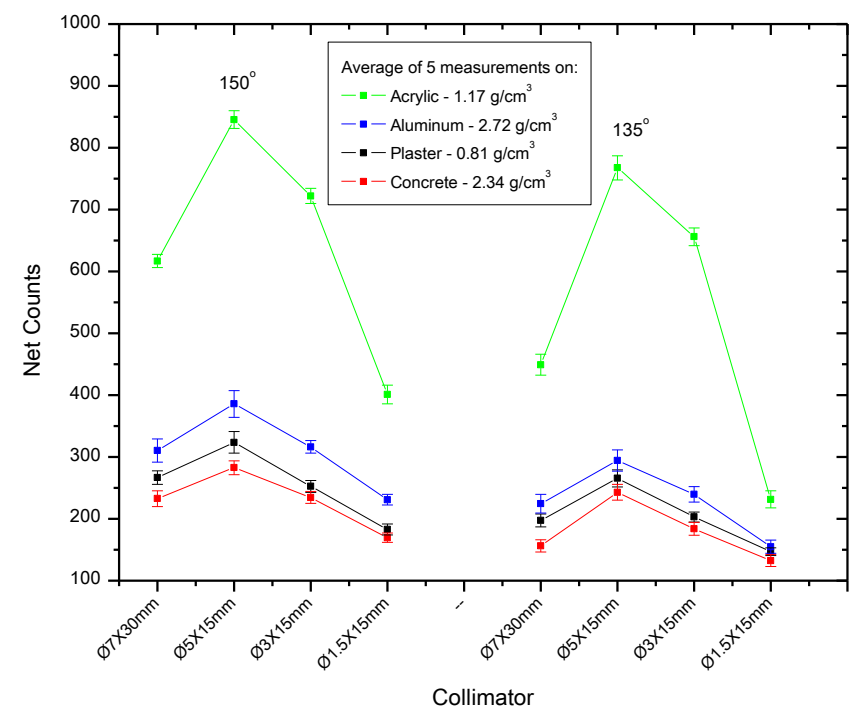

Figure 2. Net counts obtained with the four collimators tested $(\emptyset 7 \times 30 \mathrm{~mm}, \emptyset 5 \times 15 \mathrm{~mm}, \emptyset 3 \times 15$ $\mathrm{mm}$ and $\emptyset 1.5 \times 15 \mathrm{~mm})$ in two scattering angles $\left({ }^{\circ} 5^{\circ}\right.$ and $\left.150^{\circ}\right) .1000 \mathrm{~s}$ measurement time.

The greatest counts were obtained for the angle of $150^{\circ}$. These results are easily understood by considering the Klein-Nishina differential cross section that predicts a larger scattering probability in this angle at the used incident energy [2]. It is worth noting that the process is strongly affected by attenuation. The greater counts were obtained in the case of acrylic, which have the second-lowest density among the materials considered in this study. However, this low density is what results in the weakest attenuation. Plaster and concrete give similar scattering profiles despite their different densities. Further, it was verified that the collimators made of aluminum are not sufficiently long to efficiently attenuate the scattered beam.

The scattering intensities are plotted as a function of the position of the plaster sample (with the $\varnothing 10 \mathrm{~mm}$ rod) in Fig. 3. The counts decrease when the inspection volume intercepts the steel bar that has a higher density than plaster. This result indicates that the attenuation effect of the incident and scattered beams within the denser material (steel) is preponderant in this interaction. This is due to the low gamma energy produced by the ${ }^{241} \mathrm{Am}$ source $(59.54 \mathrm{keV})$. The scan performed with the scattering geometry corresponding to an angle of $135^{\circ}$ resulted in better contrast $(\mathrm{DC}=49.8 \%)$ than that performed with the geometry corresponding to an angle $150^{\circ}(\mathrm{DC}=32.8 \%)$ because of the increase in the inspection volume for greater angles. The approximate diameter of the steel rod can be directly obtained from experimental data through the Gaussian fit FWHM value. The result $\left(\mathrm{FWHM}_{135}=8.21 \mathrm{~mm}\right)$ is slightly lower than the nominal value of the bar diameter. The distance between two adjacent minima may give the distance between two bars. 


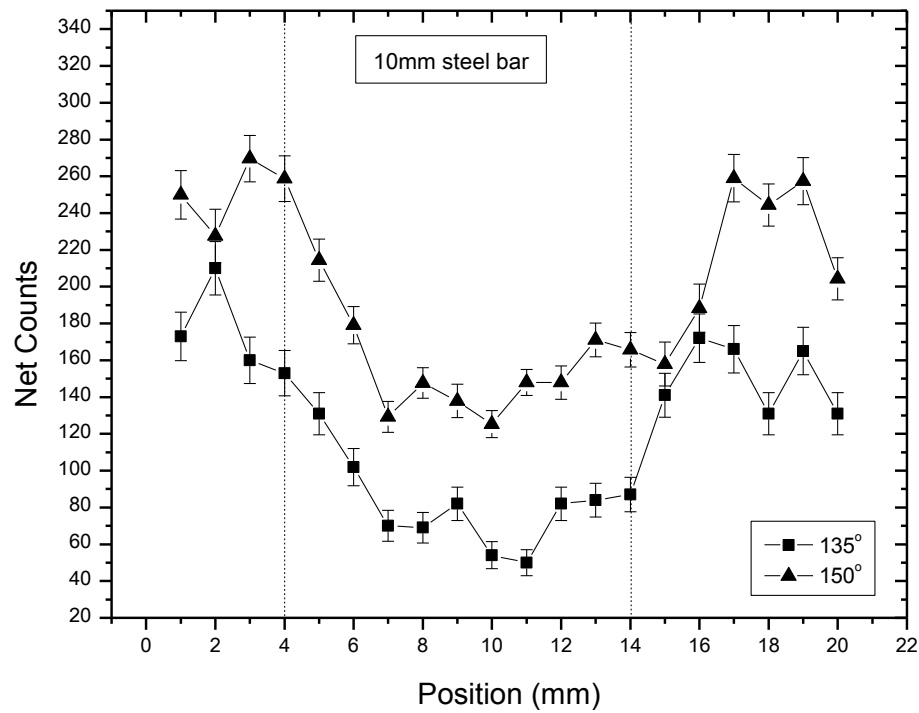

Figure 3. Net counts of backscattered photons as a function of the position of the plaster sample with a $\varnothing 10 \mathrm{~mm}$ steel bar. Scan performed at the centre of the bar, $1000 \mathrm{~s}$ measurement time at each point, $1 \mathrm{~mm}$ step.

Five scans were performed at different depths in the plaster sample in which one $\varnothing 8 \mathrm{~mm}$ steel bar was embedded, as schematized in Fig. 4 (left). The two scans that do not intercept the bar show only the fluctuations in the statistical count caused by the non-homogeneity inside the plaster. As the scans progress toward the interior of the material, the attenuation of the scattered beam causes a decrease in contrast.
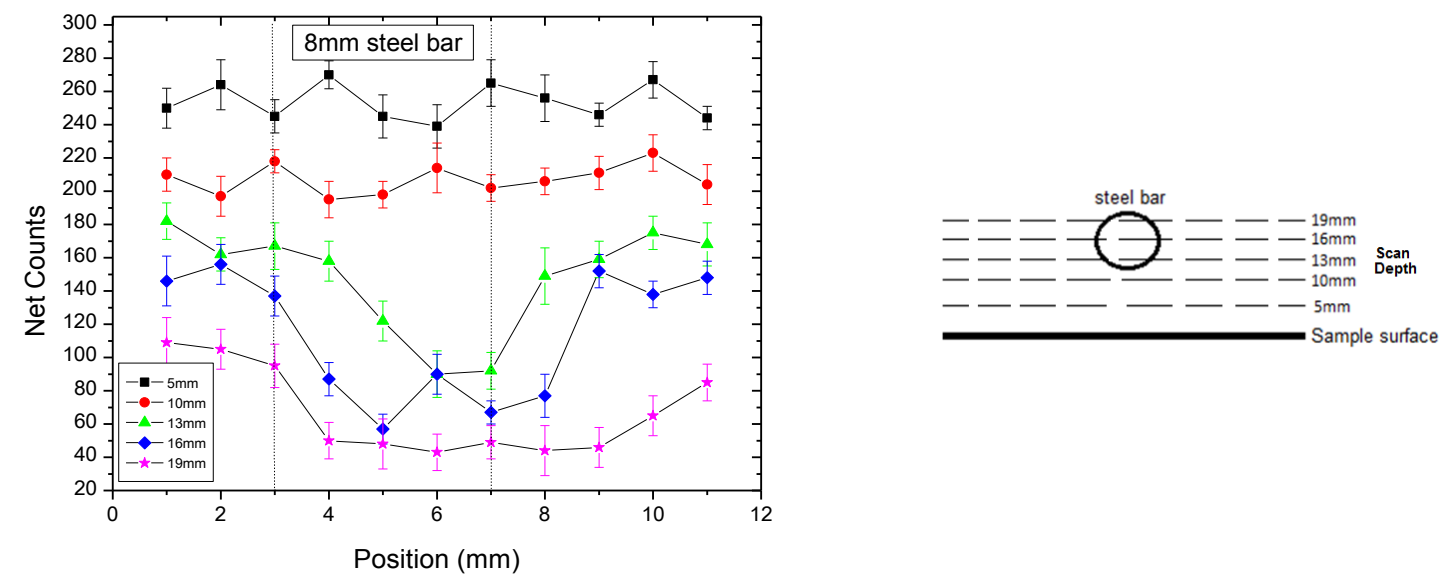

Figure 4. Net counts as a function of depth (left). Positioning scheme of the scans (right). Sample of plaster with the inclusion of $\emptyset 8 \mathrm{~mm}$ steel bar $-1000 \mathrm{~s}$ of measurement time for each point, $2 \mathrm{~mm}$ step. 
The decrease in the density contrast toward the inner portions of the sample may be used to determine the depth of an inclusion, as illustrated in Fig. 5 (left). The points in this figure represent the density contrast as a function of depth for a plaster block in which steel bars are embedded up to depths of $8 \mathrm{~mm}, 12 \mathrm{~mm}, 18 \mathrm{~mm}$, and $24 \mathrm{~mm}$ from the surface. Contrast decrease with depth up to the saturation thickness. Even though this curve has been obtained by a single preliminary analysis, it may be used to calibrate the system with the chosen configuration.
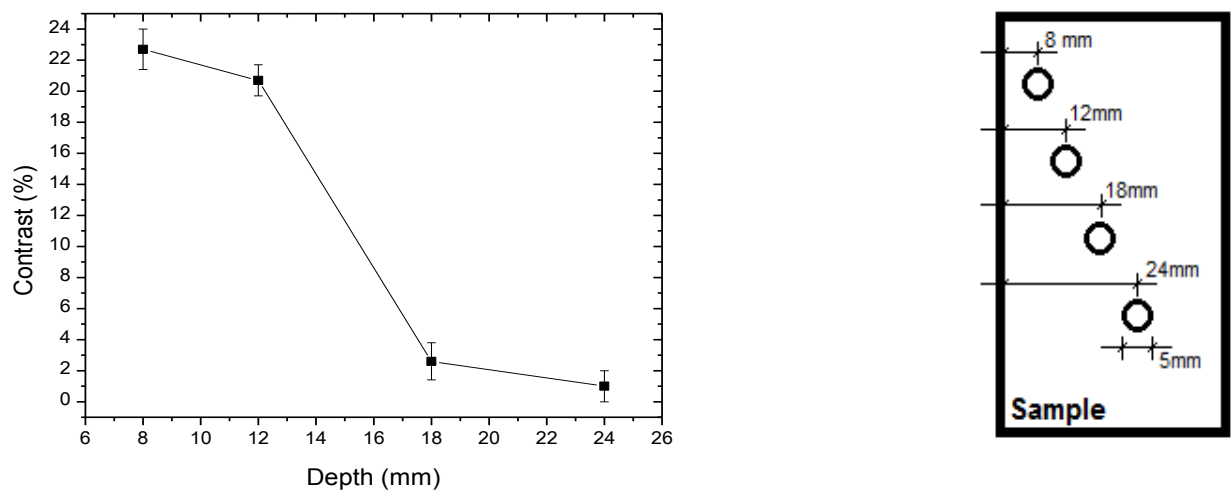

Figure 5. Contrast as a function of depth to a sample of plaster containing inclusions of steel bars of $\emptyset 5 \mathrm{~mm}$, in four different depths: $8 \mathrm{~mm}, 12 \mathrm{~mm}, 18 \mathrm{~mm}, 24 \mathrm{~mm}$ (left). Schematic drawing of the sample (right).

\section{Conclusions}

Amongst the variables studied in this work, the geometry that offers the best configurations for the construction of a lightweight and compact device for detecting superficial inclusions by employing the Compton scattering technique is one in which the source is perpendicular to the sample, the scattering angle is $135^{\circ}$, and the collimator measures $\emptyset 7 \times 30$ $\mathrm{mm}$. Even though the scattering angle of $150^{\circ}$ results in higher counts, the angle of $135^{\circ}$ angle results in the best contrast for detecting inclusions with $\varnothing 8 \mathrm{~mm}$ and $\varnothing 10 \mathrm{~mm}$ steel bars. Density contrast measures may be used to resolve the depths of inclusions in materials. The approximate position and size of inclusions may be directly assessed from the intensity versus position curves.

\section{Acknowledgments}

The authors of this work would like to thank CNPq for monetary support provided through edict MCT/CNPq 14/2008.

\section{References}

[1] M. J. Anjos, R. T. Lopes and J. C. Borges, Scattering of Gamma-Rays as Surface Inspection Technique, Nucl. Instr. and Meth. A, 280 (535) 1989.

[2] M. J. Cooper, P. E. Mijnarends, N. Shiotani, N. Sakai and A. Bansil, X-Ray Compton Scattering, Oxford University Press, New York 2004. 